\title{
IMPACTO INSTITUCIONAL DE UMA FUNDAÇÃO DE APOIO A HOSPITAL PÚBLICO UNIVERSITÁRIO
}

$\mathrm{N}$ o âmbito dos hospitais públicos universitários e das entidades universitárias públicas de forma geral, a necessidade de aprimoramento da qualidade dos serviços prestados à sociedade e da ampliação da captação de recursos financeiros para o seu aperfeiçoamento funcional é constante. Tais entidades desempenham relevante papel social, através da prestação de serviços de alta especialização e complexidade e respondem por uma demanda expressiva que lhes exige crescentes investimentos. Entretanto, a rigidez de seus orçamentos e as limitações de recursos para prover tais necessidades dificultam a atuação dos seus gestores e colocam em risco, até mesmo, a continuidade de seus serviços. Esse cenário estimula a busca de formas alternativas de gestão e vem abrindo espaço para o surgimento de grande número de fundações de apoio, fundadas por pessoas físicas e jurídicas, vinculadas às instituições universitárias, com o objetivo de apoiar atividades de ensino, pesquisa, extensão de serviços à comunidade e de desenvolvimento institucional. Essas fundações são, geralmente, instituições de direito privado, sem qualquer finalidade lucrativa, que cooperam com as entidades públicas na captação de recursos e flexibilização orçamentária, constituindo um modelo de gestão em parceria.

O Hospital das Clínicas da Faculdade de Medicina de Ribeirão Preto da Universidade de São Paulo (HCFMRP) e a Fundação de Apoio ao Ensino, Pesquisa e Assistência do Hospital das Clínicas da Faculdade de Medicina de Ribeirão Preto da Universidade de São Paulo - FAEPA, vivenciam esse modelo de gestão em parceria há pouco mais de 14 anos.

O Hospital das Clínicas da FMRP é uma entidade autárquica, com personalidade jurídica e patrimônio próprio, vinculada administrativamente à Secretaria de Estado da Saúde do Governo do Estado de São Paulo e associada à Universidade de São Paulo para fins de ensino, pesquisa e prestação de serviços à comunidade.

Trata-se de um hospital público universitário, que está inserido, desde 1989, como referência em nível terciário, na estrutura de atendimento do Sistema Único de Saúde, que representa uma referência regional para a população mais carente de uma região do Estado de São Paulo, com aproximadamente 3 milhões de habitantes, à qual o hospital faculta o acesso aos mais modernos e sofisticados procedimentos diagnósticos e terapêuticos, envolvendo tecnologia de ponta. Representa, portanto, um centro médico de excelência capaz de prover atendimento médico com elevado grau de especialização e qualidade, especialmente para as camadas sociais menos favorecidas, além de oferecer infra-estrutura para o desenvolvimento de atividades de pesquisa, bem como para formação e aperfeiçoamento para profissionais médicos e paramédicos.

Em que pese seu caráter de entidade autárquica, com autonomia administrativa e financeira, o Hospital das Clínicas mantém estreitas relações funcionais com a Faculdade de Medicina de Ribeirão Preto, cuja ampla interface pode ser visualizada em sua própria estruturação administrativa. Seu Conselho Deliberativo é composto, predominantemente, por docentes de áreas clínicas da Faculdade de Medicina, indicados pela Congregação da Faculdade, e presidido pelo seu Diretor. O regulamento do Hospital prevê que a Diretoria Clínica seja coordenada por um docente da FMRP. Do mesmo modo, a coordenação de clínicas, laboratórios e serviços médicos do Hospital das Clínicas é exercida por docentes da referida faculdade. E ainda que não haja uma exigência regimental nesse sentido, a Superintendência do Hospital, até os dias atuais, sempre foi exercida por um docente da Faculdade de Medicina.

A principal fonte de receita financeira do hospital, prevista no seu Regulamento, é a dotação orçamentária anual que recebe do Governo do Estado, estando a aplicação de seus recursos, tanto orçamentários quanto extra-orçamentários, vinculada aos órgãos de controle financeiro do Governo do Estado.

A Fundação de Apoio ao Ensino, Pesquisa e Assistência do Hospital das Clínicas da Faculdade de Medicina de Ribeirão Preto, da Universidade de São Paulo - FAEPA, é uma entidade de direito privado, sem fins lucrativos, que foi instituída em 31 de Agosto de 1988, tendo por finalidade colaborar, pelos meios 
adequados, com pessoas e entidades interessadas no desenvolvimento das Ciências da Saúde, em especial, com o Hospital das Clínicas da FMRPUSP. É regida por estatuto próprio, seu Regimento Interno, e pelas normas do Código Civil Brasileiro, sendo legalmente vedada a alteração de sua natureza, assim como a supressão dos seus objetivos primordiais. E, no caso de sua extinção, seu patrimônio destinar-se-á ao HCFMRP.

A Diretoria da FAEPA é composta por um Diretor Executivo e um Diretor Científico, com mandato de quatro anos, permitida apenas uma recondução. Os membros da Diretoria são designados pelo Conselho de Curadores, após escolha feita pelo Superintendente do Hospital das Clínicas, a partir de uma lista tríplice, elaborada pelo próprio Conselho de Curadores da Fundação. Tal como ocorre no Hospital das Clínicas, os cargos de diretoria da FAEPA têm sido ocupados por docentes da Faculdade de Medicina de Ribeirão Preto-USP.

Desde sua criação, a FAEPA vem desenvolvendo um conjunto de atividades destinadas, prioritariamente, ao aprimoramento funcional do Hospital das Clínicas, utilizando recursos obtidos de fontes variadas, dentre as quais se destacam os serviços prestados por força de convênios firmados com instituições públicas e privadas. E todos os seus recursos estão vinculados ao atendimento de suas finalidades estatutárias.

Não obstante a possibilidade, prevista em seu estatuto, de colaborar com pessoas e entidades interessadas, em seu sentido mais amplo, no desenvolvimento das Ciências da Saúde, a FAEPA mantém um relacionamento mais estreito com o Hospital das Clínicas e com a Faculdade de Medicina de Ribeirão Preto. A formalização desse relacionamento é definida com base em instrumentos jurídicos que estabelecem detalhadamente as normas, direitos, deveres e atribuições de todos os partícipes.

A ampliação das fontes de recursos financeiros do Hospital das Clínicas e a flexibilização na aplicação dos seus recursos extra-orçamentários representaram os mais importantes benefícios da utilização do adotado modelo de gestão em parceria. O gerenciamento, pela Fundação, dos recursos gerados através do atendimento médico oferecido ao Sistema Único de Saúde, a partir de 1994, representou, para o HCFMRP, um incremento significativo no montante de recursos disponíveis para a manutenção de suas atividades. Registre-se que, nos anos de 1993 e 1994, os recursos orçamentários do Hospital eram equivalentes a, aproximadamente, 42 milhões de reais por ano. No ano 2000, foram da ordem de 85 milhões de reais. Em contrapartida, em 1994, primeiro ano de gerenciamento de recursos SUS pela FAEPA, o montante total era de aproximadamente 14 milhões de reais. No ano 2000, o valor atingido foi 67 milhões e, em 2001, 75 milhões de reais. O crescimento ocorreu, principalmente, como resultado do aumento da receita originária do atendimento prestado ao SUS. Em um primeiro momento, o acréscimo foi decorrente do aumento efetivo do volume total de atividades desenvolvidas e, mais recentemente, do maior atendimento de alta complexidade, resultante de melhor organização do sistema, com hierarquização e atribuição aos hospitais universitários dos procedimentos mais sofisticados.

Em que pese o aumento da receita global, houve, no mesmo período, uma elevação expressiva dos custos de manutenção e modernização da estrutura hospitalar. Por ser um hospital geral universitário, a instituição enfrenta, naturalmente, um elevado nível de exigência, para disponibilização de métodos diagnósticos e terapêuticos mais complexos e sofisticados, nas diferentes especialidades médicas. Além disso, às necessidades inerentes a um hospital universitário aliaram-se aquelas associadas à hierarquização do atendimento mais complexo no SUS, o que tornou mais difícil a manutenção do equilíbrio econômico-financeiro do Hospital das Clínicas da FMRP-USP. A manutenção de suas estruturas complexas, que exige volumes crescentes e constantes de investimentos e um padrão de qualidade compatível com suas importantes atribuições sociais: pesquisa, assistência à população e formação de recursos humanos especializados na área médico-hospitalar, tornou indispensável a busca de recursos adicionais.

Existe, na sociedade, demanda significativa por serviços médicos de alta qualidade e complexidade, oferecidos pelos profissionais vinculados às instituições universitárias públicas. Assim, para conseguir manter seus profissionais integralmente dedicados à instituição, não os perdendo para a iniciativa privada, o HCFMRP mantém uma estrutura física, cuja destinação foi definida na década de 50, específica para atendimento de consultas e internações de pacientes pagantes pelos médicos docentes da FMRP. É uma área, totalmente independente daquela utilizada para prestação de serviços ao Sistema Único de Saúde. Portanto, entre os benefícios da aplicação desse modelo de atendimento deve ser incluída a fixação de profissionais de elevado nível de capacitação nos hospitais universitários. Na Faculdade de Medicina de 
Ribeirão Preto da USP, o atendimento a pacientes particulares por docentes, durante período limitado de tempo, mediante autorização exarada em portaria reitoral, tem sido fundamental para manter docentes clínicos em um regime de tempo integral geográfico, evitando que esses profissionais, altamente especializados, em cuja formação foram investidos recursos públicos, mudem seu regime de trabalho para 12 ou 24 horas semanais.

Desde 1994, a administração dos recursos advindos desses atendimentos vem sendo feita pela FAEPA. E desde 1999, esse atendimento foi estendido também a pacientes vinculados a planos de saúde. Os órgãos deliberativos do HCFMRP e da FAEPA tiveram o cuidado de definir que essa ampliação de atendimento não poderia ser feita com qualquer redução na qualidade ou no volume do atendimento prestado ao SUS, bem como deveria ser realizada com os recursos gerados pelo próprio atendimento. Além disso, definiu-se que os benefícios financeiros dela decorrentes deveriam ser estendidos a todos os pacientes do Hospital das Clínicas. Essa decisão foi respaldada por termo de ajuste de conduta, firmado entre a FAEPA, o HCFMRP e o Ministério Público.

Embora esse atendimento remonte à criação do Hospital das Clínicas, o fato de tratar-se de uma instituição pública, atrai críticas, principalmente sustentadas em alegações de que tal modelo de atendimento criaria uma fila dupla, em que de um lado estariam os pacientes do SUS, submetidos a enormes filas de espera pelo atendimento, e, de outro, os privados, para os quais as filas seriam menores. Esse argumento, a rigor, representa uma falácia. As filas de espera do sistema de atendimento público apenas expressam a crônica carência de recursos em face da grande demanda de pacientes necessitados. Os hospitais que prestam atendimento ao SUS não têm autonomia ilimitada para aumentar seu volume de atendimento; eles estão submetidos a tetos financeiros pactuados periodicamente. O Sistema Único de Saúde, além de não cobrir integralmente os custos dos serviços prestados, limita o número de procedimentos a serem cobertos. A própria existência do sistema privado de convênios, que atende 40 milhões de brasileiros, é resultante dessa situação. Fossem os recursos públicos suficientes para prover a assistência universal, prevista na Constituição, o sistema privado não teria essa dimensão. $\mathrm{Na}$ experiência vivida no HCFMRP, a alegação de que o aumento no volume de atendimento de pacientes vinculados a planos de saúde prejudicaria o aten- dimento aos pacientes do SUS não se sustenta quando cotejada com os dados que mostram que, nos últimos 4 anos, o hospital aumentou todos os seus índices de atendimento do SUS, incluindo uma ativação de 111 leitos (+17\%), graças, predominantemente, aos recursos gerenciados pela FAEPA. Ademais, apesar de ter ampliado seu atendimento a pacientes privados, a partir de 1999, o HCFMRP foi escolhido pelos usuários do SUS, em avaliação nacional de qualidade do atendimento, conduzida pelo Ministério da Saúde e que incluiu 4.239 hospitais, como um dos dez melhores hospitais do país, no ano 2001.

A experiência acumulada em nossa instituição demonstra que, embora o volume de recursos auferidos com tal atendimento ainda seja pequeno (um milhão e seiscentos mil reais em 2001), relativamente ao total de recursos gerenciados pela citada fundação, esses recursos são importantes para a implementação de uma série de programas de valorização de recursos humanos e de aprimoramento do hospital.

$\mathrm{O}$ gerenciamento de recursos extra-orçamentários do Hospital das Clínicas, pela FAEPA, tem sido fundamental para o investimento em custeio e modernização da instituição. Tais recursos respondem por, aproximadamente, dois terços dos gastos com material de consumo, e quase pela totalidade dos investimentos em material permanente e obras, e por $15 \%$ dos custos com manutenção e conservação. Em decorrência da redução de jornada de trabalho de servidores do Hospital das Clínicas, da abertura de novas áreas de atendimento e da necessidade de novos tipos de profissionais, a FAEPA iniciou, em 1994, um processo de contratação de empregados para suprir as necessidades da instituição. A maior parte dessas contratações envolveu servidores do próprio hospital, que foram contratados em complementação de jornada. Essas contratações aumentaram progressivamente, ao longo dos anos, atingindo aproximadamente $25 \%$ do total de servidores do hospital. O número total de empregados contratados pela Fundação para atender às diversas necessidades do HCFMRP e da FAEPA era de 1547 , ao final de 2001.

O impacto desse investimento no atendimento médico-hospitalar resultou em notável aprimoramento da assistência à saúde da comunidade mais carente, o que pode ser constatado pelos indicadores de volume de atendimento, implantação de novas tecnologias, modernização de equipamentos, volume de procedimentos mais complexos, aprimoramento da estrutura física do hospital, bem como por programas vol- 
tados para a humanização do atendimento prestado aos pacientes. Entre 1993 e 2000, o HCFMRP registrou acréscimos equivalentes a $6,2 \%, 6,7 \%, 49,7 \%$, $29,2 \%, 95,5 \%, 8 \%, 23,4 \%$ e $70 \%$, respectivamente, nos números de novos atendimentos, total de consultas, exames complementares, cirurgias, cirurgias ambulatoriais, internações, total de leitos e leitos de terapia intensiva. Apenas para referência, no ano 2000, essas atividades somaram, respectivamente, 24.652, 524.574, 2.505.571, 14.386, 8.185, 34.075, 817 e 46. Esse aumento não foi apenas quantitativo. As pesquisas para avaliação da qualidade, empreendidas pela Administração do Hospital, foram confirmadas pelo reconhecimento dos usuários do SUS, expresso através do já mencionado prêmio de qualidade auferido no ano 2001, que o incluiu entre os dez melhores hospitais do país vinculados ao SUS.

Paralelamente às atividades de investimento no custeio e manutenção do Hospital, já explicitadas, a FAEPA vem desenvolvendo um conjunto de programas voltados para a valorização de recursos humanos e de apoio a atividades de ensino e pesquisa, bem como de aprimoramento de recursos humanos. No primeiro grupo, estão programas que beneficiam todos os funcionários do HCFMRP e da FAEPA, incluindo a distribuição de vales-alimentação, de prêmio por assiduidade, de auxílio natalidade, de cestas básicas e de Natal, de uma gratificação especial de Natal, além da implementação de um programa de atendimento especializado em saúde. O segundo grupo é centrado no Programa de Auxílios FAEPA, que inclui várias modalidades de apoio: aprimoramento de recursos humanos (participação em cursos e eventos técnicos ou científicos), atividades de pesquisa e divulgação tecnológica ou científica, organização de cursos e eventos científicos ou técnicos e programas de aprimoramento da infra-estrutura institucional. Trata-se, este último, de um programa abrangente, democrático e ágil, que vem mostrando um aumento progressivo ao longo do tempo: entre 1994 e 2001, ele cresceu de 341 a 1841, no número de beneficiários, e de $\mathrm{R} \$ 100.000,00$ para $\mathrm{R} \$$ $770.000,00$, no volume de investimento. Destaque-se que esse programa beneficia, além do Hospital das Clínicas e da FAEPA, a todos os Departamentos (básicos e clínicos) da Faculdade de Medicina; inclui apoio a alunos de graduação e pós-graduação, funcionários de diferentes níveis, médicos contratados, residentes, aprimorandos e docentes da Faculdade de Medicina. Apenas para referência, no ano 2001, 300 alunos de graduação ou pós-graduação, 600 funcionários de Ní- vel Médio e Superior (não médicos), além de 500 médicos residentes ou contratados, receberam apoio para participarem de cursos ou eventos, algo que, há uma década e meia, seria difícil de imaginar. Foram, ainda, alocados recursos para apoiar a organização de 54 eventos científicos, coordenados por docentes da Faculdade de Medicina, e 98 projetos de pesquisa, desenvolvidos no hospital e na faculdade. Esse apoio inclui, também, as atividades acadêmicas e científicas desenvolvidas pelo Centro Acadêmico Rocha Lima, dos alunos do Curso Médico da FMRP-USP, bem como pelas suas diversas Ligas de Assistência, que, no ano de 2002, envolveram 27 projetos, com investimentos da ordem de $\mathrm{R} \$ 40.000,00$.

A FAEPA mantém, também, um programa de contratação de docentes colaboradores, com qualificação para ensino, pesquisa e extensão de serviços à comunidade, para desenvolvimento de atividades comparáveis às do corpo docente da Faculdade de Medicina dentro do Hospital das Clínicas. O projeto resultou na ampliação de programas e abertura de novas áreas de atuação médica no âmbito do HCFMRP, contemplando a contratação de 20 docentes colaboradores, o que representa um acréscimo aproximado de $12 \%$ em relação ao total de docentes clínicos da FMRP-USP

Entre as atividades desenvolvidas pela FAEPA, que têm apresentado um crescimento expressivo nos últimos anos, está o gerenciamento de recursos originários de convênios e contratos com instituições públicas ou privadas na área da saúde. Incluem-se, nesse grupo, aquelas que visam: 1) ao fortalecimento do Sistema Único de Saúde (programa de medicamento de alto custo do Ministério da Saúde, termos aditivos da Secretaria Estadual de Saúde); 2) ao gerenciamento de projetos de pesquisa multicêntricos, vinculados à indústria farmacêutica; 3) à implantação de Programa de Saúde da Família (coordenado pela Faculdade de Medicina de Ribeirão Preto - USP, em convênio firmado com a Secretaria Estadual de Saúde e Prefeitura Municipal de Ribeirão Preto); 4) ao gerenciamento de recursos de projetos especiais de pesquisa do CNPq (PADCT III/Milênio; Rede TB: estratégias integradas para o estudo e controle da tuberculose no Brasil).

O desenvolvimento de todos esses projetos somente é possível graças ao gerenciamento direto de recursos financeiros, pela FAEPA. Esse é, certamente, um dos principais benefícios desse modelo de parceria. Desde que sejam obedecidos os limites esta- 
belecidos nos instrumentos reguladores de cada programa, tal modelo representa, efetivamente, um mecanismo eficiente de descentralização administrativa que traz grande flexibilidade gerencial. Por um lado, a flexibilidade aumenta a agilidade na aplicação dos recursos, ao mesmo tempo em que propicia respostas institucionais mais rápidas a situações imprevistas, que não são incomuns no gerenciamento de atividades hospitalares. Por outro, esse modelo de gestão implica em um conjunto de responsabilidades para os gestores da parceria. A primeira delas é a participação ativa na captação e ampliação do volume de recursos financeiros, o que, no presente caso, em relação ao atendimento SUS, é coordenado pelo Superintendente do Hospital. A Diretoria da FAEPA responsabiliza-se pelo gerenciamento e captação de recursos vinculados ao atendimento de pacientes particulares e conveniados, bem como por fontes adicionais de receita, como ocorre com o Centro de Convenções, que foi construído para incorporar novos recursos a serem investidos no Hospital das Clínicas.

Ao mesmo tempo, a gestão descentralizada de recursos incorpora um conjunto de responsabilidades relacionadas à adequada, laboriosa e transparente prestação de contas de cada programa gerenciado; à correta aplicação dos recursos dentro das normas previstas nos instrumentos legais, que regulam os diferentes programas; além de responsabilizar, pessoalmente, seus gestores pelos atos relativos ao exercício da função gerencial.

Entretanto, não obstante todos os benefícios observados no modelo de gestão em parceria, adotado entre o Hospital das Clínicas e a FAEPA (sua fundação de apoio), as fundações de apoio vinculadas às instituições públicas universitárias, de forma geral, têm sido alvo de freqüentes questionamentos. Não se pode deixar de considerar, como fator determinante disso, a existência de um amplo desconhecimento, na sociedade, quanto à forma de atuação dessas fundações, bem como dos efetivos benefícios sociais por elas proporcionados. Quando se congregam a esses elementos fatores de natureza política e preocupações relativas aos limites da interação entre interesses públicos e privados, criam-se condições para que prosperem posições conflituosas e radicais, que não contribuem para uma avaliação adequada e profunda desse modelo de gestão em parceria público-privado. Ainda que se reconheça a possibilidade de que, eventualmente, esse modelo de gestão possa ser utilizado de modo inadequado, em prejuízo do interesse públi- co, ao mesmo tempo, é inaceitável a tentativa de, liminarmente, considerá-lo como uma forma de privatização de entidades públicas ou de burlar o regime jurídico vigente.

Deve-se sempre ter presente, quando se fala em entidades fundacionais, que elas são instituições legalmente condicionadas ao estrito cumprimento de seus objetivos estatutários, cuja não observância inviabiliza sua continuidade, pois que as sujeitam à interveniência do Ministério Público, podendo, inclusive, ser extintas por descumprimento das finalidades que justificaram sua instituição. Elas não possuem, portanto, a liberdade ilimitada, que muitos imaginam, para aplicação de recursos. E qualquer avaliação justa do modelo de parceria ora exposto deve contemplar as peculiaridades a ele inerentes e analisar seus resultados com base em fatos ou dados reais e não apenas em hipóteses ou presunções simplistas.

Existem diversos mecanismos de controle sobre a aplicação de recursos públicos, que têm condições efetivas de garantir que o modelo de gestão em parceria público-privado, adotado entre as entidades educacionais e as fundações de apoio, não prejudique o interesse social que representam. No caso do Hospital das Clínicas e da FAEPA, no plano interno, o HCFMRP e a Faculdade de Medicina exercem um acompanhamento contínuo das atividades da FAEPA, uma vez que o Conselho Curador da Fundação, que é seu órgão deliberativo máximo, é presidido pelo Diretor da Faculdade de Medicina, que é também presidente do Conselho Deliberativo do Hospital. E a maioria dos membros do dito Conselho Curador é composta por docentes que representam os Departamentos Clínicos da Faculdade, sendo que a maior parte deles é escolhida pela sua Congregação. Além disso, compõem o Conselho Curador o Superintendente e o Diretor Clínico do Hospital, o Diretor da Escola de Enfermagem da USP, um representante dos funcionários do Hospital das Clínicas e outro, dos alunos da Faculdade de Medicina. No plano externo, a FAEPA presta contas, ainda, ao seu Conselho Consultivo, composto por eminentes personalidades da sociedade civil; ao Tribunal de Contas do Estado de São Paulo; ao Ministério Público do Estado, através da Curadoria de Fundações, que é legalmente investida do dever de velar pelas entidades fundacionais, fiscalizando todos os seus atos, e que, inclusive, vem aprimorando sua atuação na tomada de contas, mediante a adoção de um relatório de atividades informatizado, mais abrangente e complexo, que passará a ser avaliado de 
maneira centralizada por uma equipe específica de técnicos do Ministério Público do Estado, finalmente, ao Instituto Nacional de Seguridade Social, como exigência para obtenção e manutenção de seu Atestado de Entidade de Assistência Social. Além desses mecanismos de controle, cada programa específico da FAEPA tem regras próprias que envolvem os diferentes participantes.

No caso, ainda, da FAEPA, seus relatórios anuais, que são elaborados com grande detalhamento, são disponibilizados integralmente na rede mundial de computadores, em seu site (www.faepa.br). A fundação também contrata, anualmente, uma auditoria externa independente, que analisa seu balanço, seus procedimentos gerenciais e seus mecanismos de controle interno.

Mostra-se patente, portanto, a existência de efetivos controles das atividades das fundações de apoio, seja em razão da sua característica de entidade de natureza social, seja por manter convênios com entidades públicas, controles que podem garantir a completa transparência do definido modelo de gestão em parceria, assim como a constante verificação do atendimento ao interesse público.

Mas o sucesso da gestão descentralizada de recursos, inerente a esse modelo de parceria, depende de um gerenciamento financeiro eficiente, associado a mecanismos internos de controle bastante rígidos, sustentados por programação cuidadosa de investimentos e responsável aplicação dos recursos gerenciados. A inobservância dessas diretrizes poderá resultar em desequilíbrio econômico-financeiro da FAEPA, colocando em risco sua viabilidade. Assim, é fundamental que, por um lado, os órgãos internos de controle exerçam ativamente suas funções estatutárias. Por outro, cabe aos gestores da fundação a implementação de sistemas de informações gerenciais eficientes, que permitam o gerenciamento individualizado de suas múltiplas atividades e da correspondente aplicação de recursos financeiros.

E, para que esse modelo de gestão possa ser implementado de modo harmonioso e eficiente, é im- portante, também, que exista uma interação adequada entre os gestores das instituições parceiras. Não obstante o relacionamento interinstitucional possa ser bastante abrangente, não se pode perder de vista que cada instituição tem suas características jurídicas e responsabilidades sociais próprias, estando seus representantes legais sujeitos às normas e regulamentos específicos da instituição autônoma que legalmente representam. As responsabilidades individuais dos gestores são intransferíveis. E, desde que haja compreensão e respeito aos limites legais existentes, a interação entre os parceiros não compromete de modo algum a eficiência de tal modelo de gestão. Por outro lado, eventuais interferências de gestores de uma instituição sobre outra podem gerar conflitos prejudiciais ao sucesso da parceria.

Os elementos aqui apresentados, ainda que de modo excessivamente resumido, demonstram que o mencionado modelo reúne qualificações para produzir grandes benefícios sociais, uma vez que representa mecanismo de flexibilização e descentralização administrativa, que se mostrou bastante benéfico na experiência relatada. O envolvimento institucional na captação e ampliação de recursos financeiros é outro aspecto bastante positivo da parceria.

A busca da excelência nos hospitais públicos universitários envolve, necessariamente, um grande esforço no sentido de ampliar as fontes e o volume de recursos financeiros, que são essenciais para cobrir seus elevados custos de manutenção. Na experiência ora apresentada, a gestão em parceria, envolvendo um hospital público universitário e uma fundação de apoio de direito privado, mostrou-se um mecanismo viável para atingir o objetivo proposto.

\section{Benedito Carlos Maciel ${ }^{1}$; Maria Aparecida de Almeida Leal Wichert ${ }^{2}$; Umbelina Scapin Próspero² \& Silvana Pischiottin Peroni ${ }^{3}$ \\ 1Docente. Departamento de Clínica Médica da Faculdade de Medicina de Ribeirão Preto - USP, Diretor Executivo da Fundação de Apoio ao Ensino, Pesquisa e Assistência do Hospital das Clínicas FMRP-USP (FAEPA). ${ }^{2}$ Advogada; Assessora Jurídica da FAEPA. ${ }^{3}$ Economista; Coordenadora Técnica-Administrativa da FAEPA.}

